\title{
Correlación: fuerza de tronco y composición corporal de hombres de Bogotá
}

\author{
Alix Johana Uscátegui Ciendua* \\ Jaime Orlando Rodríguez Peña** \\ Adriana Rocío Gutiérrez Galvis ${ }^{* * * *}$
}

Recibido: julio 15 de 2019 • Aceptado: septiembre 27 de 2019

\section{Resumen}

La investigación estableció la correlación de la fuerza de tronco en flexión y la composición corporal en hombres de Bogotá. El estudio fue realizado en el Centro de Formación en Actividad Física y Cultura del sena y participaron voluntariamente 41 hombres entre los 18 y 30 años $(m=23.44+5.81)$. Estudio cuantitativo, descriptivo, transversal y correlacional. Para la evaluación de la composición corporal se aplicó el método de análisis de impedancia bioeléctrica (BIA), con la báscula TANiTA Fit Scan BC-585F loración de la fuerza abdominal se utilizó el equipo Torso Check ${ }^{\circledR}$ (Moreau, Green, Johnson y Moreau, 2001); finalmente, para el análisis estadístico

"Artículo de investigación, con financiación del Centro de Actividad Física y Cultura - Sena, Bogotá, Colombia. Citar como: Uscátegui, A., Rodríguez, J. y Gutiérrez, A. (2019). Correlación entre la fuerza de tronco y composición corporal en hombres adultos en Bogotá. Revista de Investigación Cuerpo, cultura y movimiento, 9(2), 75-86. DOI: https://doi.org/10.15332/2422474x/5361

** Magíster en Actividad Física para la Salud, Grupo de investigación en Actividad Física y Deporte - Sena, Bogotá, Colombia. Correo electrónico: uscategui@sena.edu.co / orcID: 0000-0001-9827-3776

**** Magíster en Fisioterapia de la Actividad Física y el Deporte, líder Sennova del Servicio Nacional de Aprendizaje (Sena), Grupo de investigación en Actividad Física y Deporte - Sena, Bogotá, Colombia, Línea de investigación Entrenamiento de la fuerza, Bogotá, Colombia. Correo electrónico: Jorodriguezp@sena.edu.co / ORCID: 0000-0001-8951-9690

***** Magíster en Fisiología, líder de investigación del Servicio Nacional de Aprendizaje (Sena), Grupo de investigación en Actividad Física y Deporte - Sena, Bogotá, Colombia, Línea de investigación Entrenamiento de la fuerza, Bogotá, Colombia. Correo electrónico: argutierrez@sena.edu.co / ORCID: 0000-0002-8010-7128 
se utilizó el software SPSS $^{\circledR}$ (versión 24.0 IBM para Windows). Se empleó la prueba de correlación de Pearson para establecer las relaciones entre las variables del estudio; se fijó el nivel de significancia de $\mathrm{p}<0.05$. Los resultados obtenidos indican que existe una correlación negativa moderada que se evidencia mediante el porcentaje de masa grasa y la fuerza de flexión del tronco. Esto señala que a mayor porcentaje graso menor fuerza abdominal en flexión.

Palabras clave: resistencia flexional, entrenamiento de resistencia, músculos abdominales, obesidad abdominal. 


\section{Correlation: trunk strength and body composition of men from Bogotá}

\section{Abstract}

The research established the correlation of trunk flexion strength and body composition in men from Bogotá. The study was conducted at the SENA Physical Activity and Culture Training Center, 41 men between the ages of 18 and $30(\mathrm{~m}=23.44+5.81)$ voluntarily participated. Quantitative, descriptive, cross-sectional and correlational study. The bioelectric impedance analysis (BIA) method was applied to assess body composition, using the TANITA Fit Scan BC-585F ${ }^{\circledR}$ scale; the assessment of abdominal strength used Torso Check ${ }^{\circledR}$ equipment (Moreau, Green, Johnson and Moreau, 2001); finally, the SPSS $^{\circledR}$ software (version 24.0 Iвм for Windows) was used for statistical analysis. Pearson's correlation test was used to establish the relationships between the study variables; the significance level of $\mathrm{p}<0.05$ was set. Results obtained indicate that there is a moderate negative correlation that is evidenced by the percentage of fat mass and the trunk flexion strength. This indicates that the higher the fat percentage, the lower abdominal strength in flexion.

Keywords: flexion strength, resistance training, abdominal muscles, abdominal obesity. 


\section{Introducción}

El fortalecimiento de los músculos abdominales es de gran importancia en el rendimiento físico (Zemková, Cepková, Uvacek y Šooš, 2017), específicamente en la salud (Bianco et al., 2015). Estudios recientes concuerdan en que se debe conservar el mínimo nivel de fuerza y resistencia muscular de la zona del tronco (rectoabdominal, oblicuos externos e internos, y el transverso abdominal), para soportar la estabilización adecuada en el esqueleto axial y proporcionar así el equilibrio eficiente entre los movimientos de los miembros inferiores y superiores al momento de la ejecución de actividades deportivas o recreativas, y acciones de la cotidianidad (Kraemer y Ratamess, 2004). Un cinturón abdomino-lumbar débil puede causar desórdenes musculoesqueléticos tanto en la columna vertebral como en los miembros inferiores y el piso pélvico (Aly, ElMohsen y Hafez, 2017; Sánchez, 2004).

La fuerza disminuye con la edad, debido en gran parte a la inactividad física y las patologías asociadas con los desbalances musculares causados por la disminución de la masa muscular o pérdida del tono. La fuerza, como capacidad condicional, le permite al ser humano desplazarse de un lugar a otro y realizar actividades sencillas del quehacer diario y depende principalmente del desarrollo del sistema osteomuscular; la fuerza se define como "la capacidad para superar una resistencia externa, a través de la contracción muscular" González (2002). Esta capacidad, al igual que las demás, se desarrolla utilizando como medio la actividad física sistemática. Un estudio demostró que después de seis semanas de entrenamiento de la fuerza, se evidencian avances significativos en los consumos de glucosa en reposo en pacientes diabéticos tipo 1 y 2 (García Manso, 1999).

Desde esta perspectiva, este estudio evidenció la necesidad de correlacionar la variable de fuerza y la composición corporal. Esta última hace referencia al contenido de agua, proteínas, minerales, grasa y residuos en el organismo, y la relación directa entre la masa magra y la masa grasa (George, Fisher y Vehrs, 1996). Dentro de este componente están inmersos factores determinantes relacionados con lo genético, hereditario, nivel de actividad física, estado nutricional, factores hormonales, entre otros.

Según la Organización Mundial de la Salud (oms, 2016), la inactividad física es el cuarto factor de mortalidad en el mundo, lo que ha desencadenado sobrepeso y obesidad en la población en general. En Colombia, según la 
última Encuesta Nutricional de Salud, 1 de cada 3 adultos tiene sobrepeso, y 1 de cada 5 tiene obesidad (Ministerio de Protección Social, 2015). Varios estudios con intervenciones directas han evidenciado adolescentes con síndrome metabólico, lo que cuadruplica el riesgo de diabetes tipo 2, con más prevalencia en la adultez (Sun et al. 2008; Freedman, Dietz, Srinivasan y Berenson, 1999). En edades tempranas, etapa en la que hoy se observan altas tasas de obesidad, se han identificado casos de exceso de grasa corporal desde el inicio del proceso de gestación, lo cual está directamente relacionado con infantes que presentan diabetes tipo 2 (Ministerio de Salud de Chile, 2009; Reilly et al., 2005).

La acumulación de grasa corporal disminuye la funcionalidad de algunos segmentos corporales y ocasiona efectos negativos para la salud. El sobrepeso, la obesidad y la redistribución inadecuada de la masa grasa están vinculados con enfermedades cardiovasculares, diabetes tipo 2, hipertensión y colesterol elevado (Oliveira, Freitas, Moura, Luz Junior y Cabral, 2015; Peacock et al., 2015).

El exceso de grasa corporal en los individuos afecta negativamente la condición física y metabólica, esto ha originado trastornos a nivel general en todos los sistemas, desde la niñez hasta la vida adulta. Estudios sugieren que, si no hay intervenciones orientadas a prevenir y reducir la obesidad, tendremos adultos con enfermedades asociadas con la hipertensión arterial, hiperinsulinemia, dislipidemia y diabetes mellitus tipo 2 (Bastos, González, Molinero y Salguero del Valle, 2005). De igual modo, el exceso de grasa corporal está vinculada con el $60 \%$ de las muertes causadas por las enfermedades crónicas no trasmisibles (Clavijo, 2009). El objetivo de los programas debe ser lograr la restauración de la homeóstasis cardiovascular, musculoesquelética y metabólica (Stein y Colditz, 2004; Hills, King y Armstrong, 2007).

La sarcopenia o pérdida de la masa y funcionalidad muscular están asociadas con la inactividad física (Jackman y Kandarian, 2004). Un estudio realizó una intervención que incluyó a 60 escolares obesos de ambos sexos, de 8 a 13 años, en la ciudad de Santiago Chile. Dicha intervención incluyó ejercicio físico (entrenamiento de fuerza tres veces por semana), durante tres meses. Los resultados evidenciaron un impacto positivo de un programa de ejercicio físico de entrenamiento de fuerza en la reducción de la grasa corporal, el síndrome metabólico y los factores de riesgo cardiovascular, 
apoyando el uso del ejercicio como tratamiento para la obesidad y el aumento o mantenimiento de la masa libre de grasa (Vásquez et al., 2014).

Dado lo anterior, el presente estudio hipotetiza que algunas variables de la composición corporal pueden afectar la funcionalidad de los músculos del tronco. Por consiguiente, tiene como objetivo establecer la correlación entre las variables de fuerza de tronco en flexión y la composición corporal en hombres de Bogotá y, a su vez, responder a la siguiente pregunta de investigación: ¿existe una relación entre la fuerza del abdomen y la composición corporal en hombres adultos en Bogotá, Colombia?

\section{Metodología}

El presente corresponde a un estudio descriptivo transversal de alcance correlacional, cuyo propósito fue analizar la relación entre la masa grasa y la fuerza del tronco. Fue realizado en el laboratorio de investigación del Centro de Formación en Actividad Física y Cultura del Sena. Participaron voluntariamente 41 hombres con edades comprendidas entre 18 y 30 años, quienes firmaron un consentimiento informado y no presentaban lesiones musculoesqueléticas o neurológicas que afectaran los resultados de las evaluaciones.

\section{Procedimiento}

En la evaluación de la composición corporal se aplicó el método de análisis de impedancia bioeléctrica (BIA), con la báscula TANITA Fit Scan BC-585F . Los participantes recibieron instrucciones de no hacer ejercicio por 24 horas, tener la última comida al menos tres horas antes de la evaluación y vaciamiento de la vejiga (Yamada et al., 2017). Para realizar la valoración de la fuerza abdominal se utilizó el equipo de laboratorio Torso Check ${ }^{\circledR}$ (Moreau et al., 2001), el cual puede analizar las fuerzas del torso en las direcciones de flexión, extensión, inclinación lateral derecha, inclinación lateral izquierda, rotación izquierda y rotación derecha (Azghani, Farahmand, Meghdari, Vossoughi y Parnianpour, 2009). 


\section{Análisis estadístico}

Para el análisis de estadísticos descriptivos (media + desviación estándar [DS]) se utilizó el software SPss $^{\circledast}$ (versión 24.0 Iвм para Windows) y la prueba de distribución normal Kolmogórov-Smirnov. Se empleó la prueba de correlación de Pearson para establecer las relaciones entre las variables del estudio y se fijó el nivel de significancia de $\mathrm{p}<0.05$.

\section{Resultados}

El estudio determinó que a mayor porcentaje de masa grasa menor fuerza de flexión del tronco (tabla 1). Este hallazgo contribuye a las ciencias de la salud en dos aspectos importantes:

1. El aumento en el porcentaje de masa grasa afecta la contracción de los músculos flexores del tronco, lo que afecta la funcionalidad del core en las actividades de la vida cotidiana (Torres et al., 2010).

2. El fortalecimiento del core debe estar acompañado de estrategias que disminuyan el porcentaje de masa grasa corporal.

Tabla 1. Media (+ Ds) de los valores para las características de la población

\begin{tabular}{cc}
\hline Variable & Todos los sujetos $(\mathrm{n}=41)$ \\
\hline Edad $($ años $)$ & $23.44 \pm 5.81$ \\
Talla $(\mathrm{m})$ & $1.66 \pm 0.08$ \\
Peso $(\mathbf{k g})$ & $65.27 \pm 7.71$ \\
IMC & $23.67 \pm 3.22$ \\
Masa grasa $(\%)$ & $15 \pm 4.81$ \\
Fuerza de flexión & $194.95 \pm 52.63$ \\
(Nm) & \\
* Relación entre la masa grasa y la fuerza de flexión $\mathrm{r}=-0.407 ; \mathrm{p}=0.05$ \\
\hline
\end{tabular}

Fuente: elaboración propia. 


\section{Conclusiones}

Estudios han demostrado los efectos positivos sobre la pérdida de masa grasa, vinculando el entrenamiento de fuerza seguido de la restricción de la ingesta calórica (Hansen, Dendale, Berger, van Loon y Meeusen, 2007). Este estudio estableció la existencia de una correlación negativa moderada evidenciada entre el porcentaje de masa grasa y la fuerza de flexión del tronco. Esto indica que a mayor porcentaje graso menor fuerza abdominal en flexión.

A partir de las evidencias encontradas en la población evaluada, en la literatura existente se han constatado mejoras en los programas de intervención, lo que demostró el impacto positivo del ejercicio físico y de fuerza muscular sobre la reducción de la grasa corporal y de factores de riesgo cardiovasculares. Por esta razón y a partir de los hallazgos encontrados, se sugiere que en la población identificada con altos porcentajes de grasa corporal total y poca fuerza en el cinturón abdominal, se realice como tratamiento efectivo el uso del ejercicio (aeróbico y de fuerza muscular), y mínimo una intervención de entrenamiento de fuerza tres veces por semana (Vásquez et al., 2014). La práctica de ejercicio físico sistemático reduce el porcentaje de grasa corporal y mejora la sensibilidad a la insulina. Sin embargo, es fundamental tener en cuenta que, al suspender el ejercicio, los beneficios generados se revierten (Blair, Cheng, Holder, 2001; Cauza et al., 2005).

Por tal razón, este estudio recomienda el uso de programas como el método Pilates, el cual se ha vinculado a resultados óptimos en la ejecución de pruebas de fuerza funcional (Pastor, Nieto y Laín, 2011). El fortalecimiento del cinturón abdominal debe estar acompañado de estrategias que disminuyan el porcentaje de masa grasa corporal. Se sugiere incluir en los programas de entrenamiento un circuito intermitente con sobrecargas 3 veces por semana, entre el $62 \%$ y el $72 \%$ de una repetición máxima (RM), con una duración mínima de 8 semanas (Castrillón, Torres-Luque y de León, 2009). Esto propiciará adaptaciones a nivel de composición corporal y de fuerza-resistencia muscular general y local. 


\section{Referencias}

Aly, S., ElMohsen, A. y Hafez, S. (2017). Effect of Six Weeks of Core Stability Exercises on Trunk and Hip Muscles' Strength in College Students. International Journal of Therapies and Rehabilitation Research, 6(2), 9. DoI: https://doi. org/10.5455/ijtrr.000000237

Azghani, M. R., Farahmand, F., Meghdari, A., Vossoughi, G., y Parnianpour, M. (2009). Design and evaluation of a novel triaxial isometric trunk muscle strength measurement system. Proceedings of the Institution of Mechanical Engineers, Part H: Journal of Engineering in Medicine, 223(6), 755-66. DOI: http://doi.org/10.1243/09544119JEIM537

Bastos, A., González, R., Molinero, O. y Salguero del Valle, A. (2005) Obesidad, nutrición y Actividad Física. Revista Internacional de Medicina y Ciencias de la Actividad Física y el Deporte, 5(18), 140-53.

Bianco, A., Lupo, C., Alesi, M., Spina, S., Raccuglia, M., Thomas, E., Paoli, A y Palma, A. (2015). The sit up test to exhaustion as a test for muscular endurance evaluation. SpringerPlus, 4(1), 309. DoI: http://doi.org/10.1186/ s40064-015-1023-6

Blair, S., Cheng, Y. y Holder, J. (2001). Is physical activity or physical fitness more important in defining health benefits? Medicine and Science in Sports and Exercise, 33(Supplement), S379-S399. Dor: 10.1097/00005768-200106001-00007

Castrillón, F., Torres-Luque, G. y de León, F. (2009). Efectos de un programa de entrenamiento de fuerza sobre la composición corporal y la fuerza máxima en jóvenes entrenados. Medicina de l'Esport, 44(164), 156-62. DoI: https://doi. org/10.1016/S1886-6581(09)70126-4

Cauza, E., Hanusch-Enserer, U., Strasser, B., Ludvik, B., Metz-Schimmerl, S., Pacini, G., ... y Dunky, A. (2005). The relative benefits of endurance and strength training on the metabolic factors and muscle function of people with type 2 diabetes mellitus. Archives of physical medicine and rehabilitation, 86(8), 1527-33. DOI: https://doi.org/10.1016/j.apmr.2005.01.007

Clavijo, Z. (2009). Aspects to relationship between obesity and hypertension. The International Journal of Medicine and Science in Physical Education and Sport, 5(1):49-58. Recuperado de http://www.journalshr.com/MS-PES/papers/17/17_5.pdf

Colado Sánchez, J. C., \& Federación Española de Aeróbic y Fitness. (2004). Acondicionamiento físico en el medio acuático. Badalona: Paidotribo. 
Freedman, D., Dietz, W., Srinivasan, S. y Berenson G. (1999). The relation of overweight to cardiovascular risk factors among children and adolescents: The Bogalusa Heart Study. Pediatrics, 103(6), 1175-82. DoI: 10.1542/ peds.103.6.1175

García Manso, J. M. (2002). La fuerza: Fundamentación, valoración y entrenamiento (1. ${ }^{a}$ reimpresión). Madrid: Editorial Gymnos.

George, J., Fisher, S. y Vehrs, P. (1996). Tests y pruebas físicas. Barcelona: Ed. Paidotribo, colección fitness.

González, J. (2002). Fundamentos del entrenamiento de la fuerza. Barcelona: INDE.

Hansen, D., Dendale, P., Berger, J., van Loon, L. y Meeusen, R. (2007). The effects of exercise training on fat-mass loss in obese patients during energy intake restriction.Sports Medicine, 37(1),31-46. Dor: 10.2165/00007256-200737010-00003

Hills, A., King, N. y Armstrong, T. (2007). The contribution of physi- cal activity and sedentary behaviors to the growth and develop- ment of children and adolescents: implications for overweight and obesity. Sports Medicine, 37(6), 533-45. DOI: 10.2165/00007256-200737060-00006

Jackman, R. y Kandarian, S. (2004). The molecular basis of skeletal muscle atrophy. American Journal of Physiology-Cell Physiology, 287(4), C834-C843. DOI: 10.1152/ajpcell.00579.2003

Kraemer, W. J. y Ratamess, N. A. (2004). Fundamentals of resistance training: progression and exercise prescription. Medicine \& Science in Sports \& Exercise, 36(4), 674-88. DOI: 10.1249/01.MSS.0000121945.36635.61

Luque, G. T., García-Martos, M., Gutiérrez, C. V., y Vallejo, N. G. (2015). Papel del ejercicio físico en la prevención y tratamiento de la obesidad en adultos (The role of physical exercise in prevention and treatment of obesity in adults). Retos, 0(18), 47-51. Recuperado de https://recyt.fecyt.es/index.php/retos/ article/view/34651/18762

Ministerio de Salud Protección Social de Colombia. (2015). Encuesta Nacional de la Situación Nutricional en Colombia (Ensin) 2015. Recuperado de https://www. minsalud.gov.co/Paginas/Gobierno-presenta-Encuesta-Nacional-de-SituaciónNutricional-de-Colombia-ENSIN-2015.aspx

Ministerio de Salud de Chile. (2009). Estado nutricional de las embarazadas en control, según Región [En Línea]. Recuperado de https://www.minsal.cl/portal/url/ item/89185968d325a51ee04001011e010c92.pdf

Moreau, C., Green, B., Johnson, C. y Moreau, S. (2001). Isometric back extension endurance tests: a review of the literature. Journal of Manipulative and 
Physiological Therapeutics, 24(2), 110-22. DoI: http://doi.org/10.1067/ mmt.2001.112563

Oliveira, N. T. B. de, Freitas, S. M. S. F. de, Moura, K. F., Luz Junior, M. A. da, Cabral, C. M. N., Oliveira, N. T. B. de, ... Cabral, C. M. N. (2015). Biomechanical analysis of the trunk and pelvis during pilates method exercises: Systematic review. Fisioterapia e Pesquisa, 22(4), 443-55. Dor: https://doi. org/10.590/1809-2950/14068822042015

Pastor, T., Nieto, M. y Laín, S. (2011). Comparación de la capacidad de fuerza funcional entre tres grupos de ejercicio: participantes regulares de clases dirigidas de fitness, de método Pilates y sedentarios. Medicina de l'Esport, 46(172), 16976. DoI: https://doi.org/10.1016/j.apunts.2011.02.004

Peacock, O., Western, M., Batterham, A., Stathi, A., Standag, M., Tapp, A., Bennett, P. y Thompson D. (2015). Multidimensional individualised Physical ACTivity (Mi-PACT)-a technology-enabled intervention to promote physical activity in primary care: study protocol for a randomised controlled trial. Trials, 16(1), 381. DOI: $10.1186 / \mathrm{s} 13063-015-0892-\mathrm{x}$

Reilly, J., Armstrong, J., Dorosty, A., Emmett, P., Ness, A., Rogers, I., Steer, C. y Sherriff, A. (2005). Early life risk factors for obesity in childhood: cohort study. BML, 330(7504), 1357. DoI: https://doi.org/10.1136/bmj.38470.670903.E0

Stein, C. y Colditz, G. (2004). The epidemic of obesity. The Journal of Clinical Endocrinology \& Metabolism, 89(6), 2522-5. DOI: 10.1210/jc.2004-0288

Sun, S., Liang, R., Huang, T., Daniels, S., Arslanian, S., Liu, K, Grave,G. y Siervogel, R. (2008). Childhood obesity predicts adult metabolic syndrome: the Fels Longitudinal Study. The Journal of Pediatrics, 152(2), 191-200. DoI: 10.1016/j.jpeds.2007.07.055

Vasquez, F., Diaz, E., Lera, L., Vasquez, L., Anziani, A., y Burrows, R. (2014). Impact of a Strength Training Exercise Program on Body Composition and Cardiovascular Risk Factors in a Group of Obese Schoolchildren by Pubertal Stage. American Journal of Sports Science and Medicine, 2(1), 40-47. DoI: https://doi.org/10.12691/ajssm-2-1-8

Yamada, Y., Nishizawa, M., Uchiyama, T., Kasahara, Y., Shindo, M., Miyachi, M., y Tanaka, S. (2017). Developing and Validating an Age-Independent Equation Using Multi-Frequency Bioelectrical Impedance Analysis for Estimation of Appendicular Skeletal Muscle Mass and Establishing a Cutoff for Sarcopenia. International Journal of Environmental Research and Public Health, 14(7), 809. DOI: http://doi.org/10.3390/ijerph14070809 
Zemková, E., Cepková, A., Uvacek, M., y Šooš, L. (2017). A Novel Method for Assessing Muscle Power During the Standing Cable Wood Chop Exercise. Journal of Strength and Conditioning Research, 31(8), 2246-54. DoI: http:// doi.org/10.1519/JSC.0000000000001692 